This is a self-archived version of an original article. This version may differ from the original in pagination and typographic details.

Author(s): Walker, Simon

Title: Neural Adaptations to Strength Training

Year: 2019

Version: Accepted version (Final draft)

Copyright: @ Springer International Publishing AG, part of Springer Nature 2019

Rights: In Copyright

Rights url: http://rightsstatements.org/page/lnC/1.0/?language=en

Please cite the original version:

Walker, S. (2019). Neural Adaptations to Strength Training. In M. Schumann, \& B. R. Rønnestad (Eds.), Concurrent Aerobic and Strength Training : Scientific Basics and Practical Applications (pp. 75-86). Springer. https://doi.org/10.1007/978-3-319-75547-2_6 


\title{
Neural Adaptations to Strength Training
}

\author{
Simon Walker, PhD \\ Neuromuscular Research Center, Faculty of Sport and Health Sciences, University of Jyväskylä, Finland
}

\begin{abstract}
$\underline{\text { Abstract }}$
Scientific study of strength training has revealed numerous physiological mechanisms that contribute to: 1) acute fatigue from a single strength training session and 2) chronic adaptation to repetitive and systematic strength training. Therefore, the purpose of this chapter is initially to discuss potential neural mechanisms that influence force production from the perspective of a single repetition. Thereafter, the chapter will highlight scientific evidence for candidate neural mechanisms that acutely limit force production during a single strength training session and long-term adaptations caused by strength training. For some of these potential neural mechanisms there is strong scientific evidence and for others evidence has emerged in recent years and requires further investigation.
\end{abstract}

Keywords: motor unit, firing rate, voluntary activation, muscle activity, EMG, stimulation, V-wave, central fatigue, loading, short-term training

The purpose of this chapter is to introduce the effects of strength training on the function of the neural system. For comprehensive background knowledge concerning the structure and function of each part of the neural and muscular systems it is advisable to consult anatomy and physiology textbooks. However, as can be seen from Figure 1, muscle and nerve do not work in isolation but as an interlinked and interactive unit. The end-point of the neural system can be considered to be the neuromuscular junction (the point where motoneuron and muscle fiber are joined). The start-point of the neural system is more difficult to define but for simplicity perhaps it should be regarded as the motor cortex. Consequently, it might be preferable to think of the system as a whole and use the term "neuromuscular" rather than "neural" when discussing the acute and chronic responses to strength training in the following sections.

***Insert Figure 1 somewhere here***

Figure 1. Schematic representation of the parts of the neural system that influence force production (adapted from M oritani 2003. Chapter 3, Strength and Power in Sport).

\section{Neural effectors of force production}

The resultant force production of skeletal muscle(s) is dependent upon many different system and tissue properties, for example musculotendinous properties, such as muscle size and fiber type as well as tendon stiffness. Overall force production is naturally a sum of all the parts of the neuromuscular-tendinous system. Nevertheless, there are specific elements within the neural 
system that control and manipulate the functioning of muscle(s) for the body's specific needs at any moment in time.

Firstly, let us address the end-point of the neural system and smallest entity of the neuromuscular system, the motor unit. A motor unit consists of an alpha ( $\alpha$-)motoneuron and all of the muscle fibers it innervates. M otor units can be classified by their properties, as identified by Burke (1967), as slow (also known as Type I), fast fatigue resistant (Type Ila) or fast fatigable (Type Ilx). Consequently, the type and number of motor unit(s) activated would influence force production. Furthermore, recruitment of motor units is governed by the "size principle" (Henneman et al. 1965). This principle states that the smallest $\alpha$-motoneurons (and motor units) are recruited first and an orderly recruitment, relative to size, occurs thereafter. This would appear a good strategy since the smaller motor units are also the ones that are most difficult to fatigue and can withstand long contraction durations. When gradually increasing force production, most muscles fully recruit all motor units between 50-95\% of maximum force production (Grillner \& Udo 1971; Kulkilka \& Clamann 1981; Oya et al. 2009). During fast contractions the level of force required to recruit a specific motor unit is lowered (Desmedt \& Godaux 1978). In other words, larger (so-called high-threshold) motor units are recruited at a lower force level (i.e. more readily recruited) when the contraction is performed as fast as possible rather than during a slow contraction.

Force production is also regulated by the rate at which a motor unit is activated. In simple terms, how often an electrical discharge passes along the $\alpha$-motoneuron (i.e. motor unit action potential). This is known as firing rate (also known as discharge rate, firing frequency or rate coding). As has been clearly demonstrated during gradually increasing force contractions (so-called ramp contractions), already recruited motor units increase their firing rate as the force level increases (Milner-Brown et al. 1973; Desmedt \& Godaux 1978). Firing rate patterns appear to be reversed during fast contractions showing an initial burst of high firing rate followed by lowered rates once a certain level of force is attained (Desmedt \& Godaux 1978; Van Cutsem et al. 1998), but recruitment still follows the size principle. Differences in the interaction between motor unit recruitment and firing rate may be dependent upon the muscle in question, with small muscles seemingly more reliant on firing rate to modulate force production while large force-producing muscles rely more on recruitment (Seki \& Narusawa 1996). Nevertheless, ultimately, neural control of force production is reliant upon motor unit recruitment and firing rate whose combination determines the final signal presented to the muscle.

Prior to the $\alpha$-motoneuron, there are many upstream regulators that influence both/either the recruitment and firing rate of motor units. Afferent feedback (identified by blue lines in Figure 1) from muscular contraction influences forthcoming signals at the cortex and spinal cord. At the spinal level, afferent feedback from muscle spindles sensing stretch within muscle fibers serves to enhance (i.e. excitatory), while feedback from golgi tendon organs sensing tension within the muscle-tendon junction serve to dampen (i.e. inhibitory), signals sent to the $\alpha$-motoneuron. Also, Renshaw cells act as a negative feedback system that responds to activation of the $\alpha$-motoneuron. These regulators allow the neural system to constantly monitor and modulate force production.

Initiation of descending drive (identified by red lines in Figure 1), the signal sent down the spinal cord, originates in the cortex. A convergence of signals from the premotor cortex, cerebellum and other cortex centers influence the output of the motor cortex to the spinal cord. Various feedback 
sources contribute to the modulation of descending drive (Figure 1) during continuing contractions. A greater level of descending drive is possible through either enhanced excitation or reduced inhibition (or combination of both) within the cortex. Assuming that the effects of all other regulators of the neural system (mentioned above) are constant then a greater descending drive would lead to greater force production. It should be remembered, however, that greater descending drive may also influence the level of antagonist coactivation simultaneously (M ullany et al. 2002).

Finally, force production of agonist and synergist muscles is also affected by the level of coactivation of antagonist muscles, which may be considered as the brake applied during voluntary contraction. It is thought that coactivation is necessary to stabilize the joint for both effective movement and to act in a protective manner, and also in the case of multi-joint movement to facilitate fluid motion and coordination between muscles/joints (e.g. running and jumping). During simple single-joint movement, the level of antagonist coactivation may vary between different populations, for example, older individuals tend to demonstrate greater coactivation of antagonists than young adults (Klein et al. 2001). Despite many experimental studies, only a handful have shown evidence that strength training reduces antagonist coactivation (Carolan \& Cafarelli 1992; Häkkinen et al. 1998; Häkkinen et al. 2000; Tillin et al. 2011). Due to this uncertainty, and in the interests of brevity, the following sections will focus on modulation of agonist activation during strength training.

\section{Acute responses during a strength training session}

Following the law of specificity, it is of great importance to understand the mechanisms of acute fatigue during strength training as this/ these part(s) of the neuromuscular system are susceptible to adaptation given long-term exposure (Enoka \& Duchateau 2008). Upon reading this chapter, it should become apparent that there is a remarkable commonality between the challenged and failing parts of the neuromuscular system during a specific strength training program and their improvements over a training period. The vast majority of our (earlier) understanding on acute neuromuscular fatigue has originated from controlled experiments using electrical stimulation techniques (particularly in animals), or voluntary isometric intermittent or prolonged contractions in humans. Consulting the work of scholars such as Brenda Bigland-Ritchie, Simon Gandevia, Janet Taylor, Roger Enoka and Jacques Duchateau to name a few would help to attain knowledge from highly-controlled experiments. However, the present chapter will focus on applied scientific studies utilizing strength training typically performed in gyms.

At this point, it is perhaps prudent to outline (rather simplistically) the typical type of strength training programs studied in the literature. Maximum strength or neural strength training is comprised of high external load (>85\% 1-RM), multiple sets (3-5 per exercise) of low repetitions (1-5) and long inter-set rest periods (3-5min). Hypertrophic strength training typically uses medium external load (60-85\% 1-RM), multiple sets (2-4 per exercise) and several repetitions (8-12) and brief inter-set rest periods (1-3min). Power training aims to perform each action with the maximum possible acceleration but the external load (0-80\% 1-RM), number of sets (2-6 per exercise) and repetitions (2-10), and inter-set rest period (2-6min) can vary greatly. The names of each type of strength training are derived from the primary goals of each program. 
In terms of studying the acute effects of strength training on neural processes, the majority of studies have utilized surface EMG. The inherent weaknesses of this methodology make identifying the mechanism(s) of acute fatigue inconclusive (as explained by Farina and colleagues 2014), but it is one of the simplest and least invasive methods available. To the author's knowledge, the first studies investigating neuromuscular fatigue during strength training were performed by Häkkinen in the 1990s with athletes as subjects (Häkkinen 1993; Häkkinen 1994). Here, both M aximum strength (20 sets of 1 rep at 100\% 1-RM) and Hypertrophic (10 sets of 10 reps at 70\% 1-RM) training sessions led to decreases in maximal strength ( $\sim 24 \%$ vs $\sim 47 \%$ in men and in $\sim 21 \%$ vs. $\sim 29 \%$ women, respectively) immediately after the session and both led to decreased EMG amplitude (with the exception of Maximum strength in women for some unknown reason). One interesting study that compared three work-matched but different types of strength training was performed by McCaulley et al. (2009). Large acute decreases in maximal strength occurred during Maximum strength and Hypertrophic sessions, but EMG amplitude only decreased during the Maximum strength session. Power sessions specifically influence initial EMG amplitude (Linnamo et al. 1998), as discussed later. The lack of reduced EMG amplitude during Hypertrophic sessions is a common observation, especially in untrained/non-athlete populations (Izquierdo et al. 2009; Walker et al. 2012).

This has led to some interpreting the data that fatigue within the neural system does not occur during Hypertrophic sessions, and that the cause of the reduced strength is situated purely within the muscle. However, when inspecting changes in the frequency component of the EMG signal, as well as the amplitude, it becomes apparent that changes do occur (Izquierdo et al. 2009; GonzalezIzal et al. 2010; Walker et al. 2012). Hypertrophic strength training leads to a reduced median frequency, whereas Maximum strength training does not (as depicted in Figure 2A, Walker et al. 2012). Since median frequency is purported to represent the average conduction velocity of the firing motor units (Solomonow et al. 1990), the data might be interpreted as demonstrating maintained motor unit recruitment but reduced firing rate during Maximum strength sessions. $\mathrm{A}$ more complex situation to interpret is the lowered EMG median frequency during Hypertrophic sessions (Figure 2A).

There are several hypotheses for this phenomenon, one being slowing of the conduction velocity of the action potential due to fatigue/damage within the muscle. One other possibility for the reduced median frequency could be that there was greater synchronization in motor unit recruitment (Weytjens \& Van Steenberghe 1984). M otor unit synchronization has been shown to increase EMG amplitude (Yao et al. 2000). Therefore, it may be that increased motor unit synchronization and consequent increase in EM G amplitude negates the otherwise expected reduction in EM G amplitude due to reduced motor unit recruitment and/or firing rate. This speculation may be plausible given that athletes have a greater level of motor unit synchronization than untrained populations (M ilnerBrown et al. 1975; Semmler \& Nordstrom 1998). Furthermore, strength athletes have demonstrated reduced EMG amplitude during and after Hypertrophic sessions (Häkkinen 1994; Ahtiainen \& Häkkinen 2009), which (in-line with this hypothesis) may be due to an already higher level of motor unit synchronization and no great potential to further synchronize firings.

$* * *$ Insert Figure 2 somewhere here***

Figure 2. Fatigue-induced changes after a single strength training session in (A) EM G amplitude and median frequency (mean \pm SE) (Walker et al. 2012), (B) voluntary activation level (mean \pm SD) (Walker 
et al. 2013), and (C) Motor Evoked Potential (MEP) area and silent period length (mean $\pm S D$ ) (Ruotsalainen et al. 2014) during maximum isometric force production.

Nevertheless, perhaps the clearest method to demonstrate fatigue within the neural system during strength training sessions is to use (electrical or magnetic) stimulation methods. Merton (1954) used the interpolated twitch technique to demonstrate the inability of the human to maximally activate their skeletal muscles. Briefly, if superimposing a high-intensity electrical stimulation to the innervating nerve or directly to the activated muscle during a maximum voluntary contraction induces an increase in the measured force, then the muscle was not performing to its potential and the deficit was located within the neural system. This method has shown that fatigue within the neural system exists during Hypertrophic strength sessions as demonstrated by a reduction in voluntary activation level (Walker et al. 2013, Figure 2B).

Another method to detect fatigue within the neural system is to utilize Transcranial Magnetic Stimulation (TMS) directly to the motor cortex and measure the response at the muscle. In an interesting study by Ruotsalainen et al. (2014), measurement of the Motor Evoked Potential (MEP) in the EMG signal of the biceps brachii after each set of a $3 \times 10-R M$ (i.e. Hypertrophic) bicep curl session showed an initial increase concomitant to muscular fatigue after set 1 (Figure $2 \mathrm{C}$ ). It could be suggested that corticospinal excitability (i.e. the efficacy of all parts of the cortical and spinal network mentioned above) increased in order to possibly overcome the inability of the musculature to produce the required force level to sustain performance. This initial increase was then followed by a progressive decrease after each set. The decreases in MEP size set-to-set were matched by an elongation of the silent period following the stimulation (Figure $2 \mathrm{C}$ ). This likely represents fatigueinduced cortical inhibition, which could account for a reduction in descending drive following (Hypertrophic) strength training sessions. For more information regarding neural fatigue and the potential mechanisms that cause such fatigue, recent work by Carroll and colleagues (Carroll et al. 2017) is worth consulting.

Finally, to briefly address the effects of a Power training session, it is worth noting that the reductions in both force and EMG amplitude during the initial $\sim 100 \mathrm{~ms}$ of contraction were greater than the reductions of maximum and EMG amplitude (Linnamo et al. 1998). This finding was contrasted by similar reductions of all time-points during isometric action following a Maximum strength session. These findings highlight that the manifestation of fatigue during Power training, where the aim is to accelerate the load as fast as possible, specifically affect the initiation of contraction. These findings seem to give clues as to the specific training-induced adaptations from various strength training programs.

\section{Neural adaptations to strength training}

As noted in the first section of this chapter, simplistically, greater motor unit recruitment and/or firing rate of agonist and synergists would increase force production. Therefore, greater force production could be due to; 1 ) greater descending drive from the cortex and/or 2) greater spinal motoneuron excitability and/or reduced inhibition influencing descending drive (Aagaard \& 
Thorstensson 2003). In this section, studies using various methods to estimate and quantify these potential adaptations during strength training will be presented.

The amplitude of the Surface EMG signal is taken to be a gross measure of motor unit recruitment and firing rate of those motor units that are within the pick-up area of the electrodes. This has led authors to utilize surface EMG as an indication of neural adaptation to strength training. Over the past 3-4 decades many studies have shown increases in EMG amplitude during maximum force production from 8-21 weeks of strength training in various populations (Häkkinen \& Komi 1983; Narici et al. 1989; Aagaard et al. 2002; Suetta et al. 2004; Walker et al. 2016) but particularly previously untrained individuals. Also, rate of EMG rise or average EMG amplitude over the initial period of force production (e.g. $50-100 \mathrm{~ms}$ ) has been shown to increase during fast contractions (Häkkinen et al. 1985; Aagaard et al. 2002; Tillin et al. 2012). Nevertheless, use of surface EMG to infer neural adaptation is fraught with pitfalls. Specific methodological constraints and confounding factors as highlighted by Farina et al. (2014) suggest that other mechanisms other than neural adaptation could account for increases in EMG amplitude, particularly during maximum force production.

One physiological, but muscular adaptation, which could affect the EMG signal is the propagation of the action potential (Arabadzhiev et al. 2014) and another is the level of motor unit synchronization (Yao et al. 2000). This last point is interesting since strength-trained individuals have shown a greater level of synchronization than non-trained controls (M ilner-Brown et al. 1975; Semmler \& Nordstrom 1998). Whether increased motor unit synchronization aids strength development is debatable (Yao et al. 2000; Kidgell et al. 2006), but it highlights a limitation of the method in assessing neural adaptation.

Indwelling or intramuscular EM G electrodes have been used by some groups to quantify motor unit activity patterns before and after strength training. In particular, early increases ( $\varangle 6$ weeks training) in firing rate have been demonstrated in several populations during maximum (Knight $\&$ Kamen 2001; Christie \& Kamen 2010) and rapid force production (Van Cutsem et al. 1998). Furthermore, increased firing rate at the beginning of fast contractions was accompanied by improved rate of force development (Van Cutsem et al. 1998). These findings may help to explain the observed increases in EM G during the initial part of the force-time curve of fast contractions following Power training (Häkkinen et al. 1985; Aagaard et al. 2002; Tillin et al. 2012), as mentioned above.

Perhaps a more direct and non-invasive method to assess neural adaptation is peripheral nerve/muscle stimulation. The twitch interpolation technique has demonstrated increases in voluntary activation level in several muscles (Knight \& Kamen 2001; Walker et al. 2016) due to strength training, in both young and older subjects (Figure $3 \mathrm{~A}$ ). While these findings could infer greater motor unit recruitment and/or firing rate, these findings likely reflect greater firing rate given the above mentioned evidence from intramuscular EMG and that most muscles' motor units are fully recruited below $95 \%$ of maximum force.

Other studies have used peripheral stimulation to quantify H-reflex and V-wave amplitude before and after strength training. Submaximal electrical stimulation to a peripheral nerve induces an artificial reflex response recorded by surface EMG, known as the Hoffmann- or H-reflex. Conversely, a maximal electrical stimulation elicits a compound action potential (i.e. M-wave) that concomitantly abolishes the $\mathrm{H}$-reflex response in a resting condition. The $\mathrm{V}$-wave is the voluntary equivalent of the 
H-reflex, and voluntary drive must surpass the $M$-wave abolishment to be observable in the EMG signal. Hence, it is a measure of overall efferent output from the motoneuron pool (Aagaard et al. 2002) and an increase in V-wave (normalized to the maximum $M$-wave) represents an increase in descending drive. Possibly due to methodological considerations (e.g. the proximity of the muscle to the spinal cord) distal muscles, such as the triceps surae, have been utilized to demonstrate traininginduced adaptations to strength training using this method. Strength training has been accompanied by increases in V-wave responses after only 3 weeks (Vila-Cha 2012) and 14 weeks (Aagaard et al. 2002, Figure 3B) of training in healthy young subjects and in older individuals (Unhjem et al. 2015). These studies combined this method with resting $\mathrm{H}$-reflex stimulation to show that changes in spinal excitability did not occur as a consequence of training, which supports the previously stated hypothesis that neural adaptations are largely a result of supraspinal changes.

\section{$* * *$ Insert Figure 3 somewhere here***}

Figure 3. Strength training-induced increases in (A) voluntary activation level (mean $\pm S E$ ) (Knight \& Kamen 2001), (B) V-wave amplitude (mean+SD) (Aagaard et al. 2002), and (C) conditioned MEP amplitude (mean \pm SE) (Weier et al. 2012).

TMS stimulation of the motor cortex to measure neural adaptation has led to mixed findings. While Kidgell et al. (2010) and Weier et al. (2012) observed greater M EP size following training (possibly indicating greater descending drive), Lee et al. (2009) did not. In support of reduced corticospinal inhibition due to strength training, Latella et al. (2012) observed shortened silent periods in both the trained and untrained rectus femoris muscles. Furthermore, reduced intracortical inhibition was observed following 4 weeks of Hypertrophic squat training (Weier et al. 2012, Figure 3C). Therefore, although there has been limited study of corticospinal excitability/inhibition during strength training, it may be that a training-induced decrease in the level of inhibition is the most likely candidate for the improved descending drive.

Finally, it should be noted that the use of stimulation methods have also largely been limited to being induced under a constant force level, either maximal or submaximal. Therefore, the potential role of training-induced supraspinal adaptations that increase motor unit firing rate has not been investigated fully. But current evidence perhaps enables us to speculate that inhibitory mechanisms at both the supraspinal and spinal level are supressed after strength training, which allows greater descending drive and ultimately greater motor unit firing rates. These neural adaptations may well account for improved performance after short-duration strength training. Future investigations may look to other methods to assess cortical functioning during rapid contractions and new insights may be attained.

\section{Summary}

The neural system controls force production through an intricate and complex system that has many levels of control. Each element of this system is capable of increasing (i.e. facilitating) or decreasing 
(i.e. inhibiting) force output. Ultimately, the sum of these different effectors results in a specific recruitment pattern consisting of motor unit recruitment and motor unit firing rate. Increasing either or both motor unit recruitment and/or firing rate will lead to an increase in force output assuming that the muscle and tendon are able to transmit this force in a similarly effective manner. Performing a single session of strength training will challenge various elements of the neural system leading to acute modifications in the ability of the system to recruit motor units and/or limit their firing rate. Training is a repetitive stimulus that fatigues these parts of the neural system and these specifically adapt to be able to increase motor unit recruitment and/or firing rate. However, as this chapter highlights, there is still work to be done in order to fully understand which parts of the system are fatigued during a single strength training session and also which parts adapt during training.

\section{References}

Aagaard P, Simonsen EB, Andersen JL, Magnusson P, Dyhre-Poulsen P. 2002a. Neural adaptations to resistance training: changes in evoked $\mathrm{V}$-wave and $\mathrm{H}$-reflex responses. Journal of Applied Physiology 92 (6):2309-2318.

Aagaard P Simonsen EB, Andersen JL, Magnusson P, Dyhre-Poulsen P. 2002b. Increase rate of force development and neural drive of human skeletal muscle following resistance training. Journal of Applied Physiology 93 (4):1318-1326.

Aagaard P, Thorstensson A. 2003. Neuromuscular aspects of exercise-adaptive responses evoked by strength training. pp.70-106. In: Kjaer M, Krosgaard m, Magnusson P (ed). Textbook of Sports Medicine: basic science and clinical aspects of sports injury and physical activity. Wiley publishers, Chichester, England.

Ahtiainen JP, Häkkinen K. 2009. Strength athletes are capable to produce greater muscle activation and neural fatigue during high-intensity resistance exercise than nonathletes. Journal of Strength and Conditioning Research 23 (4):1129-1134.

Arabadzhiev TI, Dimitrov VG, Dimitrov GV. 2014. The increase in surface EM G could be a misleading measure of neural adaptation during early gains in strength. European Journal of Applied Physiology 114 (8):1645-1655.

Carolan B, Cafarelli E. 1992. Adaptations in coactivation after isometric resistance training. Journal of Applied Physiology 73 (3):911-917.

Carroll TJ, Taylor JL, Gandevia SC. 2017. Recovery of central and peripheral neuromuscular fatigue after exercise. Journal of Applied Physiology 122 (5):1068-1076.

Christie A, Kamen G. 2010. Short-term training adaptations in maximal motor unit firing rates and afterhyperpolarization duration. Muscle and Nerve 41 (5):651-660.

Desmedt JE, Godaux E. 1978. Ballistic contractions in fast or slow human muscles: discharge patterns of single motor units. Journal of Physiology 285 : 185-196. 
Enoka RM, Duchateau J. 2008. Muscle fatigue: what, why and how it influences muscle function. Journal of Physiology 586 (1):11-23.

Farina D, Merletti R, Enoka RM. 2014. The extraction of neural strategies from the surface EMG. Journal of Applied Physiology

Gonzalez-Izal M, Malanda A, Navarro-Amezqueta I, Gorostiaga EM, Mallor F, Ibanez J, Izquierdo M. 2010. EMG spectral indices and muscle power fatigue during dynamic contractions. Journal of Electromyography and Kinesiology 20 (2):233-238.

Grillner S, Udo M. 1971. Recruitment in the tonic stretch reflex. Acta Physiologica Scandinavia 81 (4): 571-573.

Häkkinen K, Komi PV. 1983. Electromyographic changes during strength training and detraining. Medicine and Science in Sports and Exercise 15 (6):455-460.

Häkkinen K, Komi PV, Alen M. 1985. Effect of explosive type strength training on isometric force- and relaxation-time, electromyographic and muscle fibre characteristics of leg extensor muscles. Acta Physiologica Scandinavia 125 (4):587-600.

Häkkinen K. 1994. Neuromuscular fatigue in males and females during strenuous heavy resistance loading. Electromyography and Clinical Neurophysiology 34 (4): 205-214.

Häkkinen K, Kallinen M, Izquierdo M, Jokelainen K, Lassila H, Mälkiä E, Kraemer WJ, Newton RU, Alen M. 1998. Changes in agonist-antagonist EMG, muscle CSA, and force during strength training in middle-aged and older people. Journal of Applied Physiology 84 (4):1314-1349.

Häkkinen K, Alen M, Kallinen M, Newton RU, Kraemer WJ. 2000. Neuromuscular adaptation during prolonged strength training, detraining and re-strength-training in middle-aged and elderly people. European Journal of Applied Physiology 83 (1):51-62.

Kidgell DJ, Sale MV, Semmler JG. 2006. M otor unit synchronization measured by cross-correlation is not influenced by short-term training of a hand muscle. Experimental Brain Research. 175 (4):745753.

Kidgell DJ, Stokes M A, Castricum TJ, Pearce AJ. 2010. Neurophysiological responses after short-term strength training of the biceps brachii muscle. Journal of Strength and Conditioning Research 24 (11):3123-3132.

Klein CS, Rice CL, Marsh GD. 2001. Normalized force, activation, and coactivation in the arm muscles of young and old men. Journal of Applied Physiology 91 (3):1341-1349.

Knight CA, Kamen G. 2001. Adaptations in muscular activation of the knee extensor muscles with strength training in young and older adults. Journal of Electromyography and Kinesiology 11 (6):405412.

Kulkilka CG, Clamann HP. 1981. Comparison of the recruitment and discharge properties of motor units in human brachial biceps and adductor pollicis during isometric contractions. Brain Research $219(1): 45-55$. 
Lee M, Gandevia SC, Carroll TJ. 2009. Short-term strength training does not change cortical voluntary activation. Medicine and Science in Sports and Exercise 41 (7):1452-1460.

Linnamo V, Häkkinen K, Komi PV. 1998. Neuromuscular fatigue and recovery in maximal compared to explosive strength loading. European Journal of Applied Physiology 77 (1-2):176-181.

McCaulley GO, M cBride JM, Cormie P, Hudson MB, Nuzzo JL, Quindry JC, Triplett TN. 2009. Acute hormonal and neuromuscular responses to hypertrophy, strength and power type resistance exercise. European Journal of Applied Physiology 105 (5):695-704.

Milner-Brown HS, Stein RB, Yemm R. 1973. The orderly recruitment of human motor units during voluntary isometric contraction. Journal of Physiology 230 (2):359-370.

Milner-Brown HS, Stein RB, Lee RG. 1975. Synchronization of human motor units: possible roles of exercise and supraspinal reflexes. Electromyography and Clinical Neurophysiology 38 (3):245-254.

Moritani T. 2003. Motor unit and motoneurone excitability during explosive movement. pp.27-49. In: Komi PV (ed). Strength and Power in Sport (2nd edition). Blackwell Science Ltd, Oxford, England.

Mullany H, O'M alley M, St Clair Gibson A, Vaughan C. 2002. Agonist-antagonist common drive during fatiguing knee extension efforts using surface electromyography. Journal of Electromyography and Kinesiology 12 (5):375-384.

Narici MV, Roi GS, Landoni L, Minetti AE, Cerretelli P. 1989. Changes in force, cross-sectional area and neural activation during strength training and detraining of the human quadriceps. European Journal of Applied Physiology 59 (4):310-319.

Oya T, Riek S, Cresswell AG. 2009. Recruitment and rate coding organization for soleus motor units across entire range of voluntary isometric plantar flexions. Journal of Physiology 587 (19):4737-4748.

Ruotsalainen I, Ahtiainen JP, Kidgell DJ, Avela J. 2014. Changes in corticospinal excitability during an acute bout of resistance exercise in the elbow flexors. European Journal of Applied Physiology 114 (7):1545-1553.

Seki K, Narusawa M. 1996. Firing rate modulation of human motor units in different muscles during isometric contraction with various forces. Brain Research 719 (1-2):1-7.

Semmler JG, Nordstrom MA. 1998. Motor unit discharge and force tremor in skill- and strengthtrained individuals. Experimental Brain Research 119 (1):27-38.

Suetta C, Aagaard P, Rosted A, Jakobsen AK, Duus B, Kjaer M, Magnusson SP. 2004. Training-induced changes in muscle CSA, muscle strength, EMG, and rate of force development in elderly subjects after long-term unilateral disuse. Journal of Applied Physiology 97 (5):1954-1961.

Tillin NA, Pain MT, Folland JP. 2011. Short-term unilateral resistance training affects the agonistantagonist but not the force-agonist activation relationship. Muscle and Nerve 43 (3): 375-384.

Tillin NA, Pain MT, Folland JP. 2012. Short-term training for explosive strength causes neural and mechanical adaptations. Experimental Physiology 97 (5):630-641. 
Van Cutsem M, Duchateau J, Hainaut K. 1998. Changes in single motor unit behavior contribute to the increase in contraction speed after dynamic training in humans. Journal of Physiology 513 (1):295-305.

Unhjem R, Lundestad R, Fimland MS, M osti MP, Wang E. 2015. Strength training-induced responses in older adults: attenuation of descending neural drive with age. AGE 37 (3):9784.

Vila-Cha C, Falla D, Correia M V, Farina D. 2012. Changes in H reflex and V wave following short-term endurance and strength training. Journal of Applied Physiology 112 (1):54-63.

Walker S, Davis L, Avela J, Häkkinen K. 2012. Neuromuscular fatigue during dynamic maximal strength and hypertrophic resistance loadings. Journal of Electromyography and Kinesiology 22 (3):356-362.

Walker S, Peltonen H, Avela J, Häkkinen K. 2013. Neuromuscular fatigue in young and older men using constant or variable resistance. European Journal of Applied Physiology 113 (4):1069-1079.

Walker S, Blazevich AJ, Haff GG, Tufano JJ, Newton RU, Häkkinen K. 2016. Greater gains after training with accentuated eccentric than traditional isoinertial loads in already strength-trained men. Frontiers in Physiology 7:149.

Weier AT, Pearce AJ, Kidgell DJ. 2012. Strength training reduces intracortical inhibition. Acta Physiologica 206 (2):109-119.

Yao W, Fluglevand RJ, Enoka RM. 2000. M otor-unit synchronization increases EM G amplitude and decreases force steadiness of simulated contractions. J of Neurophysiology 83 (1):441-452. 


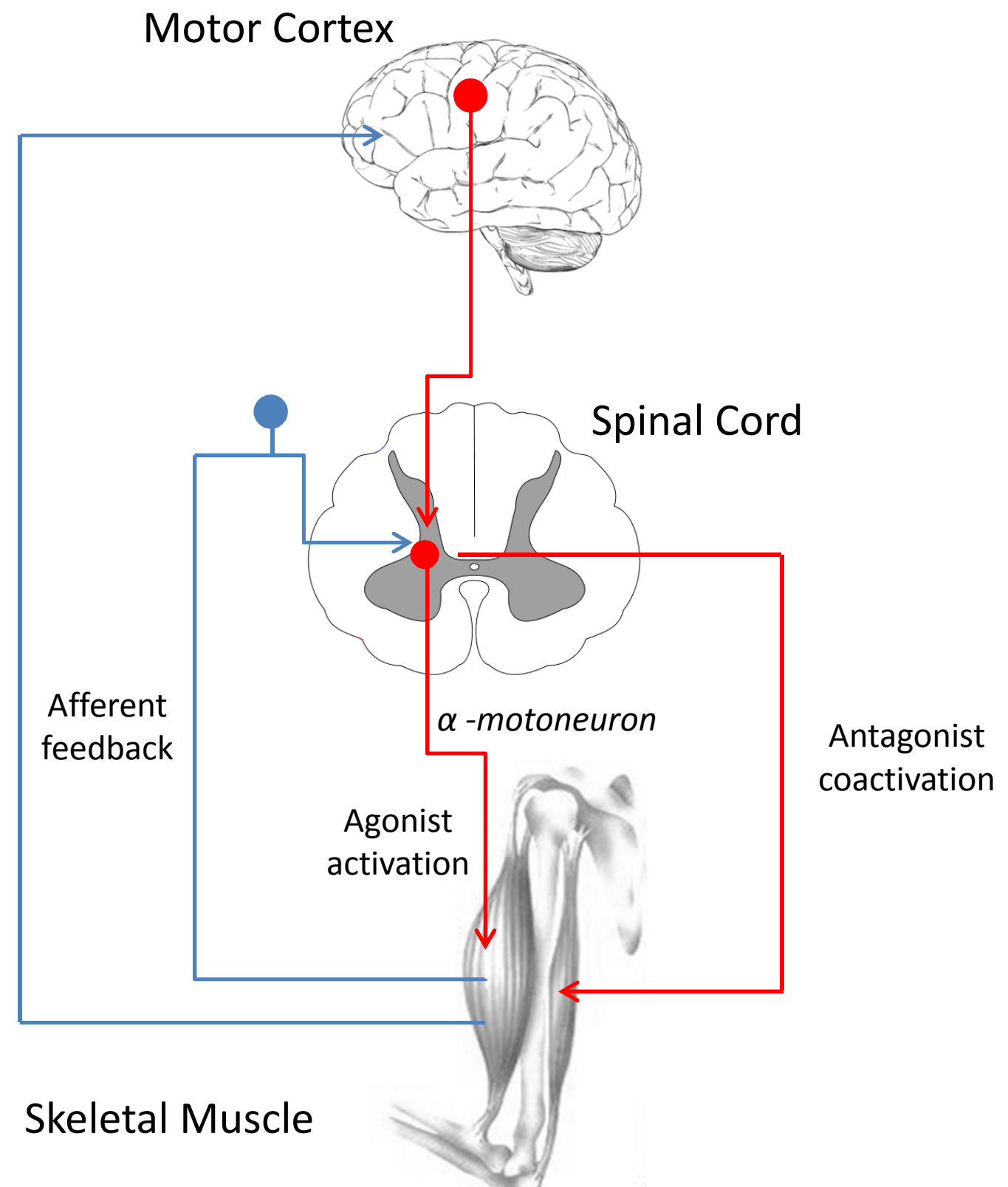



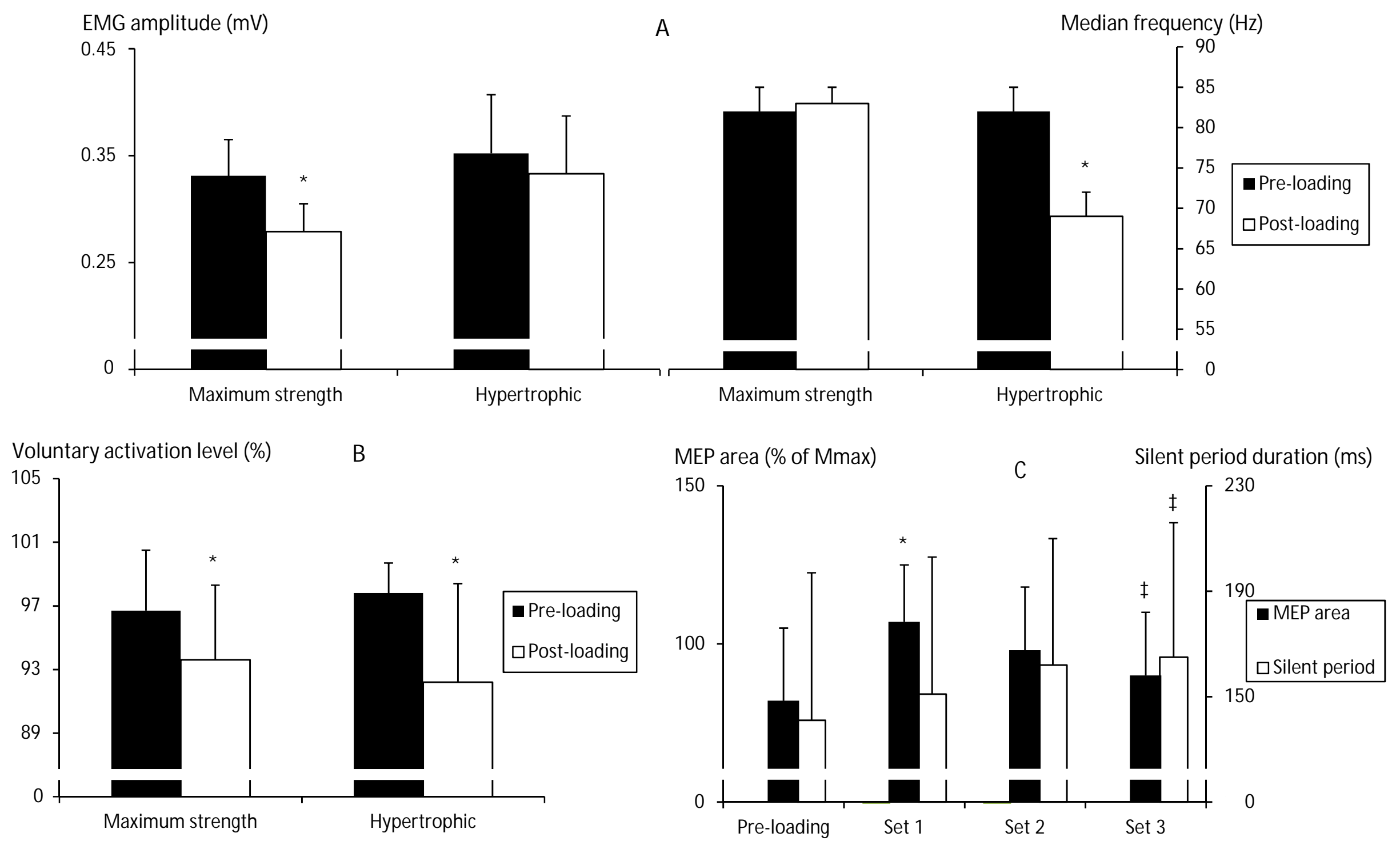
Voluntary activation level \%

100

99

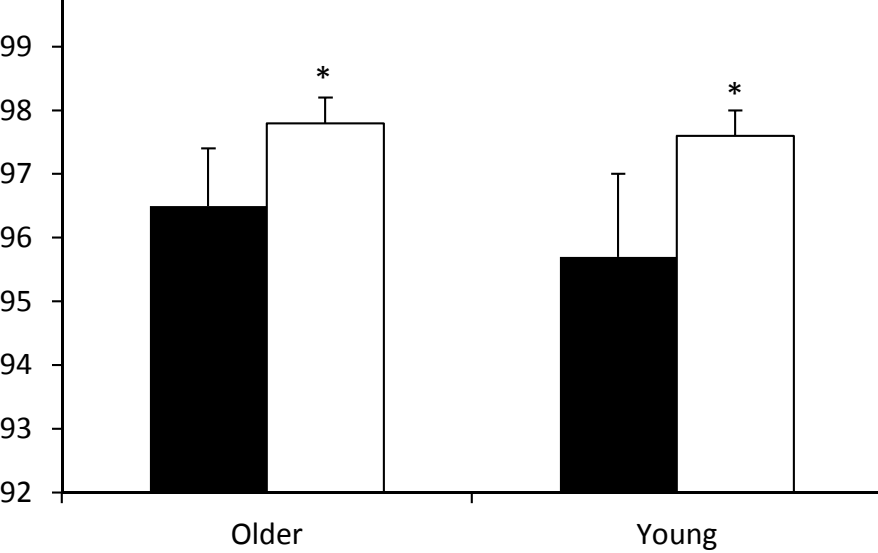

Normalized responses

(\% of maximum M-wave)

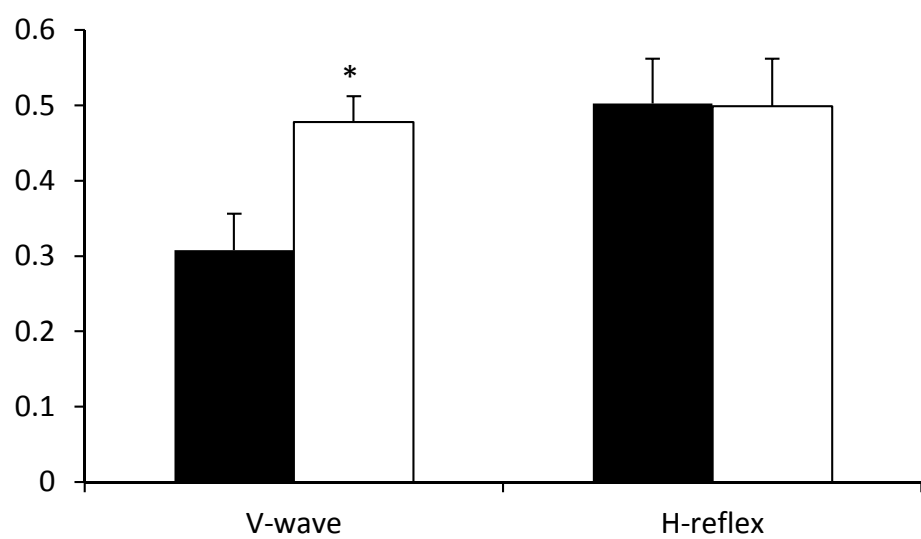

Conditioned MEP amplitude (\% resting response)

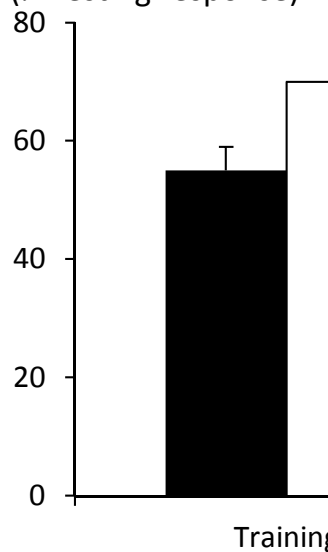

B

- Pre-training

$\square$ Post-training
- Pre-training

$\square$ Post-training 